\title{
GLOBAL TOPOLOGICAL ASPECTS OF CONTINUOUS-TIME LINEAR DYNAMICALLY VARYING (LDV) CONTROL
}

\author{
Edmond A. Jonckheere and Stephan Bohacek \\ Dept of Electrical Engineering- Systems \\ University of Southern California \\ Los Angeles, CA 90089-2563 \\ (213) $740-4457$ \\ jonckheeQeudoxus.usc.edu
}

\begin{abstract}
Linear Dynamically Varying (LDV) systems are a subset of Linear Parameter Varying (LPV) systems characterized by parameters that are dynamically modeled. An LDV system is, in most cases of practical interest, a family of linearized approximations of a nonlinear dynamical system indexed by the point around which the system is linearized. Special attention is devoted to nonlinear dynamical systems running over a Riemannian manifold. Such (local) differential geometric concepts as curvature play a crucial role in defining the LDV approximation. Furthermore, such (global) topological properties as parallelizability, Euler characteristics-and a global "flatness" concept-are crucially involved in defining the problem in a computationally attractive coordinate-dependent fashion. Finally, an LQ trajectory tracking problem is formulated, revealing a partial differential Riccati equation, itself related to a linear $\mathrm{PDO}$, for which an index theorem can be formulated.
\end{abstract}

\section{LDV Systems}

Given a differentiable $n$-D manifold $\Theta$, a dynamical system over $\Theta$ is defined by

$$
\dot{\theta}(t)=f(\theta(t)) ; \quad \theta(0)=\theta_{0}
$$

From the coordinate independent point of view, $f$ should be interpreted as a vector field, that is, a section $\Theta \rightarrow T \Theta$ through the tangent bundle $T \Theta$ with fiber $\mathbb{R}^{n}$ of the manifold $\Theta$. The solution $\theta(t)$ is viewed as a differentiable curve with its velocity defined in the tangent space

$$
\frac{d}{d t} \theta(t) \in T_{\theta(t)} \Theta
$$

Depending on the global topology of $\Theta$, it is not always possible to have an everywhere nonvanishing vector field. Such a nonsingular vector field is a section through the tangent sphere bundle and there might be obstructions to constructing the latter section. A theorem due to Hopf asserts that, for a compact, orientable, smooth manifold $\Theta, \chi(\theta)=\sum_{i}$ index $\left(\theta_{i}\right)$, where $\chi$ denotes the Euler characteristics, the $\theta_{i}$ 's the singularities of the field, and index $(\cdot)$ the Brouwer degree of the local Gauss map.

In the applications considered here, (1) is some nominal dynamics that should be enforced via a small extraneous control effort $u \in \mathcal{U} \subseteq \mathbb{R}^{p}$. To this effect, we consider a perturbed dynamical system [3]

$$
\frac{d}{d t} \tilde{\theta}(t)=\tilde{f}(\tilde{\theta}(t), u(t)) ; \quad \tilde{\theta}(0)=\tilde{\theta}_{0}
$$

such that

$$
\tilde{f}(\theta, 0)=f(\theta) ; \quad \tilde{f}(\theta, \mathcal{U}) \subseteq T_{\theta} \Theta
$$

and to be controlled such that, for $\theta_{0}$ and $\tilde{\theta}_{0}$ sufficiently close, $\lim _{t \rightarrow \infty} d(\tilde{\theta}(t), \theta(t))=0$ for some small control $u$. In the above, $\tilde{f}$ is defined as a section through the tangent bundle along the projection on the first factor map $\pi_{1}$ or equivalently a lifting of $\pi_{1}$. The latter means that the bottom right-hand corner triangle of the following diagram commutes

$$
\begin{array}{ccc}
T \Theta \times\left(\mathcal{U} \times \mathbb{R}^{p}\right) & \stackrel{(A, B)}{\longrightarrow} & T \Theta \\
\downarrow & \stackrel{\tilde{f}^{\prime}}{\longrightarrow} & \downarrow \\
\Theta \times \mathcal{U} & \stackrel{\pi_{1}}{\longrightarrow} & \Theta
\end{array}
$$

The difficulty in defining a convenient linearized tracking error is that $f(\theta)$ and $\tilde{f}(\tilde{\theta}, u)$ live in nearby, but different tangent spaces. To compare the two vectors 
$f(\theta) \in T_{\theta} \Theta$ and $\tilde{f}(\tilde{\theta}, u) \in T_{\tilde{\theta}} \Theta$ a parallel transport of $\tilde{f}$ into $T_{\theta} \Theta$ is performed. With both vectors in the same tangent space, the so-called covariant differentiation can be made. To do this parallel transport, $\theta$ is assumed to be a Riemannian manifold with fundamental metric $\langle x, x\rangle=\sum_{i j} g_{i j} x^{i} x^{j}$ and endowed with the Levi-Civita connection:

$$
\nabla: \Gamma(T \Theta) \times \Gamma(T \Theta) \rightarrow \Gamma(T \Theta)
$$

where $\Gamma(T \Theta)$ denotes the module of all sections $\Theta \rightarrow$ $T \Theta$ or vector fields over $\Theta$. The connection provides the notion of covariant differentiation of a vector field (e.g., $e_{j}$ ) along another vector field (e.g., $e_{i}$ ), defined by linearity and the Leibniz rules from $\nabla_{e_{i}} e_{j}=\sum_{k} \Gamma_{i j}^{k} e_{k}$ where $\left\{e_{i}\right\}$ is a Cartan moving reference frame in the tangent space and the $\Gamma$ 's are the Christoffel symbols of the Levi-Civita connection:

$$
\Gamma_{i j}^{k}=\frac{1}{2} \sum_{l}\left(\frac{\partial g_{j l}}{\partial x^{i}}+\frac{\partial g_{i l}}{\partial x^{j}}-\frac{\partial g_{i j}}{\partial x^{l}}\right) g^{l k}
$$

where $\left\{x^{i}\right\}$ is a system of local coordinates naturally associated with $\left\{e_{i}\right\}$ via the exponential map $[1,5.13]$. Given a curve $c:[0,1] \rightarrow \Theta$ such that $c(s=1)=\tilde{\theta}$ and $c(0)=\theta$, the vector field $\varphi$ is a parallel transport of $\tilde{f}$ along $c$ if $\nabla_{\frac{d c}{d s}} \varphi=0$ and $\varphi(s=1)=\tilde{f}$. It is customary to rewrite the operator of covariant differentiation along a curve $c, \nabla_{\frac{d c}{d s}}$, as $\frac{D}{d s}$. (Warning: $s$ is not the arc length.)

The parallel transport from $\tilde{\theta}$ to $\theta$ depends on the path joining the two points. It is claimed that if we choose the path to be the minimizing geodesic $c_{*}$, the resulting differential does not depend on the path provided it stays close enough to $c_{*}$. Indeed, there exists a neighborhood of $\theta, N_{\theta}$, such that any two points within $N_{\theta}$ can be joined by a unique minimizing geodesic. We therefore restrict $\tilde{\theta} \in N_{\theta}$. Consider two minimizing geodesic paths $c_{1}, c_{2} \subseteq N_{\theta}$ joining $\tilde{\theta}$ and $\theta$. Because of the curvature, the results of the parallel transport of $\tilde{f}$ along $c_{1}$ and $c_{2}, f_{c_{1}}$ and $f_{c_{2}}$, resp., need not be the same. Actually, in case $n=2$, the Gauss-Bonnet theorem asserts that the mismatch or holonomy angle is given by

$$
\iint_{\Sigma} K d S=\angle\left(\tilde{f}_{c_{1}}, \tilde{f}_{c_{2}}\right)
$$

where $K$ denotes the Gauss curvature, $d S$ is the surface element, and $\Sigma$ is the surface bounded by $c_{1} \cup c_{2}$. (If $n>2$, use the sectional curvature.) Since

$$
\frac{\text { area }}{\text { length }} \rightarrow 0, \tilde{\theta} \rightarrow \theta
$$

it follows that $\tilde{f}_{c_{1}}-\tilde{f}_{c_{2}}$ goes to zero faster than $\tilde{f}_{c_{i}}-f$ so that the differential does not depend on the path provided the latter be sufficiently close to the geodesic. From the above, we define the local state ${ }^{1}$ of the linearized approximation as $x=\left.\frac{d c}{d s}\right|_{s=0}$ and the following easily follows:

$$
\begin{aligned}
\frac{D \tilde{f}(c(s), 0)}{d s} & =\nabla_{\frac{d s}{d s} \tilde{f}} \\
& =\nabla_{\sum_{j} x^{j} e_{j}} \sum_{i} \tilde{f}^{i} e_{i} \\
& =\sum_{i, j} x^{j} \nabla_{e_{j}} \tilde{f}^{i} e_{i} \\
& =\sum_{i, j} x^{j}\left(\frac{\partial \tilde{f}^{i}}{\partial x^{j}} e_{i}+\sum_{k} \Gamma_{j i}^{k} \tilde{f}^{i} e_{k}\right) \\
& =\sum_{i j} e_{i}\left(\frac{\partial \tilde{f}^{i}}{\partial x^{j}}+\sum_{k} \Gamma_{j k}^{i} \tilde{f}^{k}\right) x^{j} \\
& =\sum_{i, j} e_{i}\left(F_{\theta}\right)_{j}^{i} x^{j}
\end{aligned}
$$

where $F_{\theta}$ is a mixed covariant/contravariant tensor whose components are

$$
\left(F_{\theta}\right)_{j}^{i}=\frac{\partial \tilde{f}^{i}}{\partial x^{j}}+\sum_{k} \Gamma_{j k}^{i} \tilde{f}^{k}
$$

Therefore, we get

$$
\frac{D \tilde{f}(c(s), 0)}{d s}=F_{\theta} x
$$

From the obvious relations

$$
\begin{aligned}
\dot{x} & =\nabla_{\dot{\theta}} x \\
\frac{D f(c(s))}{d s} & =\nabla_{x} \dot{\theta}
\end{aligned}
$$

it follows that, contrary to the Euclidean case, $\dot{x}$ and $\frac{D f}{d s}$ do not match, because indeed, exploiting the symmetric (torsionless) property of the Levi-Civita connection, we get

$$
\dot{x}-\frac{D f}{d s}=\nabla_{f} x-\nabla_{x} f=[f, x]
$$

The right-hand side Lie bracket induces a linear map $\operatorname{ad}_{f}: x \mapsto[f, x]$. Therefore, if we define,

$$
A_{\theta}=F_{\theta}+\operatorname{ad}_{f}
$$

we get $\frac{d}{d t} x(t)=A_{\theta(t)} x(t)$ as expected.

Furthermore, redoing the same in the product space $\Theta \times \mathcal{U}$ and using the invariance relation (3) yields

$$
\frac{d}{d t} x(t)=A_{\theta(t)} x(t)+B_{\theta(t)} u(t)
$$

${ }^{1}$ The conflict of notation that $x$ is also the local coordinate vector is not dangerous. 
where $\left(B_{\theta}\right)_{j}^{i}=\frac{\partial \tilde{f}^{i}}{\partial u^{j}},(5)$ together with (1) is called a Linear Dynamically Varying (LDV) system.

Finally, we can justify the top row of the commutative diagram (4). To be specific, $(A, B)$ induces a mapping

$$
\begin{aligned}
(A, B): \gamma & \mapsto \nabla_{\gamma} \tilde{f} \\
\Gamma\left(T \Theta \times\left(\mathcal{U} \times \mathbb{R}^{p}\right)\right) & \rightarrow \Gamma(\Theta \times \mathcal{U}, T \Theta)
\end{aligned}
$$

where $\Gamma(X, T Y)$ denotes the set of sections $X \rightarrow T Y$ along a smooth map $X \rightarrow Y$. The mapping is defined as follows. Consider a section

$$
\gamma(\theta)=\sum_{j} x^{j}(\theta) e_{j}+\sum_{j} u^{j}(\theta) \varepsilon_{j}
$$

where $\left\{e_{1}, \cdots, e_{n}, \varepsilon_{1}, \cdots, \varepsilon_{p}\right\}$ is a moving frame in $\Theta \times$ $\mathcal{U}$. The image of $\gamma$ is

$$
\begin{aligned}
(A, B) \gamma & =\sum_{j} x^{j}\left(\nabla_{e_{j}} \tilde{f}\right)+\sum_{j} u^{j}\left(\nabla_{\varepsilon_{j}} \tilde{f}\right) \\
& =\sum_{i} e_{i}\left(\sum_{j} A_{j}^{i} x^{j}+\sum_{j} B_{j}^{i} u^{j}\right)
\end{aligned}
$$

\section{2 global parallelizability issues}

Local, computationally attractive matrix representations for $A_{\theta}, B_{\theta}$ are guaranteed to exist. To obtain a global matrix representation of $A_{\theta}$, it is necessary to have a coordinate frame $\left\{e_{1 \theta}, \ldots, e_{n \theta}\right\}$ in the tangent space $T_{\theta} \Theta$, smoothly depending on $\theta, \forall \theta \in \Theta$. This is the issue of parallelizability of the manifold $\Theta$. Because a necessary condition for parallelizability is $\chi(\Theta)=0$, not all manifolds are parallelizable. However, as proved $[9$, Theorem 95], if $\Theta$ is a Lie group with a real Lie algebra, then $\theta$ is parallelizable. If $\Theta$ is not parallelizable, the guiding idea is to attempt to construct a parallelizable covering manifold, if this is possible, and then "lift" the system to the covering space.

\section{1 covering surface}

Consider a continuous-time dynamical system running on a Möbius strip $M$, to be controlled so as to track the mid circle. Draw on the strip a $\theta$-dependent reference frame: say, $e_{1}$ orthogonal to the ribbon and $e_{2}$ aligned with the ribbon. Clearly, the $e_{2}$ axis has to be "flipped" across the edge where the two cuts of the ribbon are glued. The problem with this dynamical system can be seen from the fact that the state of the controlled LDV system, as a vector in the tangent space, is continuous across the bonding; however, since the $e_{2}$ axis has to be flipped, the state component $x^{2}$ suffers a discontinuity across the bonding. To remove this difficulty, the intuitive idea is not to flip the reference frame at this stage, but allow the LDV system to run beyond the cut on another sheet, with a consistent reference frame, on top of the sheet of the first lap, and after two laps along the Möbius strip, there is no more misalignment of the axes. Mathematically, this amounts to cover the Möbius strip $M$ with a "doubly twisted" Möbius strip $\bar{M}$, which is homeomorphic to a cylinder, and is hence parallelizable.

The Möbius strip example can be put in the following broader context:

Theorem 1 If $\Theta$ is a complete surface with Gaussian curvature $\leq 0$, then it has a covering surface homeomorphic to $\mathbb{R}^{2}$. Furthermore, if $\Theta$ is a surface with constant vanishing Gauss curvature, that is, if $\Theta$ is either the cylinder, the torus, the Möbius strip, or the Klein bottle, it can be covered by $\mathbb{R}^{2}$, as shown by the following diagram in which all arrows are covering maps:

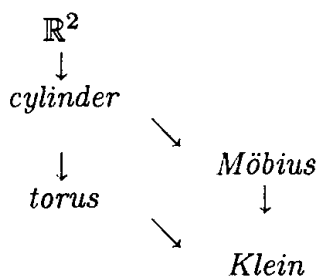

Proof See $[13$, p. 259] for first claim and $[8$, p. 420] for second claim.

A surface of constant $>0$ Gaussian curvature is not parallelizable (because by the Gauss-Bonnet theorem $\chi \neq 0$ ), nor is its standard covering, the sphere $S^{2}[8]$. For a compact surface of genus $g$ (guaranteed $[8$, p. 266] to have a point at which its curvature is $>0$ ), only the torus $(g=1)$ is parallelizable, because of the relation $\chi=2-2 g$.

\section{2 covering manifold}

Theorem 2 If $\Theta$ has a "flat" connection ${ }^{2}$, that is, if its curvature $R(\alpha, \beta)=\nabla_{\alpha} \nabla_{\beta}-\nabla_{\beta} \nabla_{\alpha}-\nabla_{\{\alpha, \beta]}=0$, then its universal covering space is parallelizable.

Proof. From $[1,5.10 .3]$, if $R=0$, the connection is locally parallelizable. By going to the covering manifold, curvature and local parallelizability are unchanged. However, the fact that the universal covering manifold is simply connected implies that its connection is parallelizable.

It can be shown that the Möbius strip can be endowed with a flat connection $[1$, p. 237].

${ }^{2}$ There is no connection between this flatness concept in terms of vanishing curvature and the "output flatness" concept of [10]. 
Corollary 3 If $\Theta$ is almost parallelizable, that is, if the structure group of its tangent bundle can be reduced to a discrete group, then $\Theta$ has a parallelizable universal covering manifold.

Proof. For indeed, an almost parallelizable manifold has a flat connection $[11$, p. 32$]$. (In fact, as easily seen, a finite covering would have made it parallelizable.) $\square$

\section{3 liftability}

Theorem 4 Let $\bar{\Theta}$ be a covering manifold of $\Theta$, let $\pi: \bar{\Theta} \rightarrow \Theta$ be the associated covering map, and let $T \bar{\Theta} \rightarrow T \Theta$ be the induced tangent bundle covering map. Then the vector field $f: \Theta \rightarrow T \Theta$ lifts to the covering manifold as depicted in the following diagram:

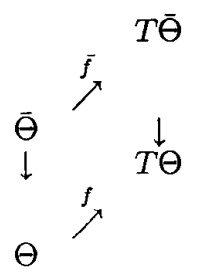

Proof: Take a point $\bar{\theta} \in \bar{\Theta}$ and let $\pi(\bar{\theta})=\theta$, where $\pi$ denotes the covering projection. By definition of the covering manifold map, there exist neighborhoods $O_{\bar{\theta}}$, $O_{\theta}$ linked by a homeomorphism $h: O_{\bar{\theta}} \rightarrow O_{\theta}$. Let

$$
\begin{aligned}
& \phi_{\theta}: O_{\theta} \rightarrow \mathbb{R}^{n}, \quad \theta \mapsto 0 \\
& \bar{\phi}_{\bar{\theta}}: O_{\bar{\theta}} \rightarrow \mathbb{R}^{n}, \quad \bar{\theta} \mapsto 0
\end{aligned}
$$

be the coordinate functions around $\theta$ and $\bar{\theta}$, respectively. The homeomorphism $h$ induces a diffeomorphism of the coordinate spaces

$$
\phi \circ h \circ \bar{\phi}_{\bar{\theta}}^{-1}: \mathbb{R}^{n} \rightarrow \mathbb{R}^{n}
$$

By definition of the tangent space $T_{\theta} \Theta$, the vector $f(\theta)$ is the equivalence class $C_{\theta}$ of all curves $c_{\theta}$ in $\mathbb{R}^{n}$ tangent at 0 . Clearly, all of the curves $\left(\phi_{\theta} \circ h \circ \bar{\phi}_{\bar{\theta}}^{-1}\right)^{-1}\left(c_{\theta}\right)$, $c_{\theta} \in C_{\theta}$ are tangent to each other at 0 in the coordinate space of $\bar{\theta}$. We define $\bar{f}(\bar{\theta})$ to be the equivalence class of $\left(\phi_{\theta} \circ h \circ \bar{\phi}_{\bar{\theta}}^{-1}\right)^{-1}\left(c_{\theta}\right)$, which is easily seen not to depend on the representative $c_{\theta}$. Next, it is easily seen that things match up properly if $\theta, \vec{\theta}$ are within two coordinate patches.

\section{LQ LDV tracking}

Clearly, if we want to track a trajectory of (1) with the system (2), the linearized tracking error will be given by the LDV system (5). Motivated by this tracking problem or by the dual synchronization problem, we define the following LQ problem:
Theorem 5 Assume that the maps $A$ and $B$ are differentiable and that $(A, B)$ is stabilizable in the sense that there exists a differentiable map $K: T \Theta \rightarrow \mathbb{R}^{p}$ such that the solution to $\dot{x}=(A+B K) x$ is stable. Then

$$
X_{\theta_{0}}\left(x_{0}, x_{0}\right)=\inf _{u} \int_{0}^{\infty}\left(\langle x(\tau), x(\tau)\rangle+\|u(\tau)\|^{2}\right) d \tau
$$

defines a positive definite, symmetric bilinear form (order 2 covariant tensor) $X \in T^{*} \Theta \otimes T^{*} \Theta$ satisfying the partial differential Riccati equation, in terms of matrix representation relative to the dual basis $\left\{e^{i}=x^{i}\right\}$,

$$
\sum_{i} \frac{\partial X}{\partial x^{i}} f^{i}+G+A_{\theta}^{T} X_{\theta}+X_{\theta} A_{\theta}-X_{\theta} B_{\theta} B_{\theta}^{T} X_{\theta}=0
$$

(a coordinate independent version also exists) and such that $A_{\theta}-B_{\theta} B_{\theta}^{T} X_{\theta}$ is stable.

Proof The proof is standard except for two thingsdifferentiability of $X_{\theta}$ and the use of the covariant differentiation. Assuming without loss of generality by stabilizability that $A_{\theta}$ is stable, a slight generalization of the concepts of [5] yields families of operators $\mathcal{A}: T \Theta \rightarrow L^{2}(\Gamma(T \Theta)), \mathcal{B}: \Theta \times L^{2}(\mathcal{U}) \rightarrow L^{2}(\Gamma(T \Theta))$ such that $x=\mathcal{A}_{\theta_{0}} x_{0}+\mathcal{B}_{\theta_{0}} u, x_{0} \in T_{\theta_{0}} \Theta$. It follows that

$$
\begin{aligned}
\int_{0}^{\infty} & \left(\langle x(\tau), x(\tau)\rangle+\|u(\tau)\|^{2}\right) d \tau \\
= & (x, x)_{L^{2}(\Gamma(T \Theta))}+(u, u)_{L^{2}(\mathcal{U})} \\
= & \left\langle x_{0}, \mathcal{A}^{*} \mathcal{A} x_{0}\right\rangle+\left(u,\left(I+\mathcal{B}^{*} \mathcal{B}\right) u\right)+2\left(u, \mathcal{B}^{*} \mathcal{A} x_{0}\right)
\end{aligned}
$$

Observe that $I+\mathcal{B}_{\theta}^{*} \mathcal{B}_{\theta}$ is a Wiener-Hopf operator if $\theta$ is an equilibrium point. Since $I+\mathcal{B}^{*} \mathcal{B}>\epsilon I$, an extension of the argument of [4] yields

$$
X_{\theta_{0}}\left(x_{0}, x_{0}\right)=\left\langle x_{0},\left(\mathcal{A}^{*} \mathcal{A}-\mathcal{A}^{*} \mathcal{B}\left(I+\mathcal{B}^{*} \mathcal{B}\right)^{-1} \mathcal{B}^{*} \mathcal{A}\right) x_{0}\right\rangle
$$

Since $I+\mathcal{B}^{*} \mathcal{B}>\epsilon I,\left(I+\mathcal{B}^{*} \mathcal{B}\right)^{-1}$ has a differential and therefore so has $X_{\theta}$. In the proof that $X$ satisfies the PDRE, the key point is that the principle of optimality implies that

$$
\int_{0}^{t}\left(\langle x(\tau), x(\tau)\rangle+\|u(\tau)\|^{2}\right) d \tau+X_{\theta(t)}(x(t), x(t))
$$

is monotone increasing with $t$ along the vector field $\tilde{f}$. Taking the covariant derivative $\frac{D}{d t}=\nabla_{\tilde{f}}$ of the above considered as a function $\Theta \rightarrow \mathbb{R}$ and setting it $\geq 0$ for all $x, u$ yields a LMI, from which, following the classical path of approach, we derive the PDRE along with the additional claims.

Remark: The stabilizability assumption here is considerably stronger than that of [2], where the stabilizing feedback is not even assumed to be continuous. The hard part in the approach of [2] is to prove that under this weak assumption the $\mathrm{LQ}$ and $H^{\infty}$ feedbacks are continuous. 
Theorem 6 The PDRE has a unique stabilizing, differentiable solution.

Theorem 7 The unique, differentiable, stabilizing solution to the PDRE, $X$, is the maximum of all solutions, in the sense that for any other solution $Y$, we have $X_{\theta}-Y_{\theta} \geq 0, \forall \theta$.

This PDRE, related to the Hamilton-Jacobi-Isaacs equation, has uniqueness of its solution secured by the stabilizability property, itself equivalent to the maximality property. This maximality property allows us to solve the equation locally, and then "stitch" together all local solutions to get the global stabilizing solution.

\section{Hamiltonian PDO}

Here we proceed in a local, coordinate dependent fashion, since the factorization $X_{\theta}=V_{\theta} W_{\theta}^{-1}$ is hard to justify intrinsically. Injecting this factorization in the PDRE yields an invariant subspace problem

$$
P_{\sigma}\left(\begin{array}{c}
W_{\theta(x)} \\
V_{\theta(x)}
\end{array}\right) \subseteq\left\{\left(\begin{array}{c}
W_{\theta(x)} \\
V_{\theta(x)}
\end{array}\right)\right\}
$$

for the partial differential operator

$$
\begin{aligned}
& P_{\sigma}= \\
& \left(\begin{array}{cc}
A_{\theta(x)}-\sum_{i} f^{i}(\theta(x)) \frac{\partial}{\partial x^{i}} & -B_{\theta(x)} B_{\theta(x)}^{T} \\
-G_{\theta(x)} & -A_{\theta(x)}^{T}-\sum_{i} f^{i}(\theta(x)) \frac{\partial}{\partial x^{i}}
\end{array}\right)
\end{aligned}
$$

The computation of $\left(\begin{array}{l}W \\ V\end{array}\right)$ can be reduced to finding eigenvalues $\lambda \in \mathbb{C}$ and sections $\left(\begin{array}{l}w \\ v\end{array}\right): \Theta \rightarrow$ $\left(\begin{array}{c}T \Theta \\ T^{*} \Theta\end{array}\right)$ in the kernel of another partial differential
operator:

$$
\left(P_{\sigma}-\lambda I\right)\left(\begin{array}{c}
w_{\theta(x)} \\
v_{\theta(x)}
\end{array}\right)=0
$$

The following theorem allows us to choose the correct eigenvalues $\lambda$ and the correct invariant subspace in the kernel of the $P_{\sigma-\lambda I}$ operator:

Theorem 8 If $\Lambda$ is chosen so that the solution to $\dot{Z}=$ $\Lambda Z$ along the trajectories of $\dot{\theta}=f(\theta)$ is such that $Z \rightarrow$ 0 , then $A_{\theta}-B_{\theta} B_{\theta}^{T} V_{\theta} W_{\theta}^{-1}$ is stable.

The operator $P_{\sigma}$ is defined, locally in the coordinate patch, as a pseudo-differential operator [12]

$$
P_{\sigma}\left(\begin{array}{c}
w_{\theta(x)} \\
v_{\theta(x)}
\end{array}\right)=\frac{1}{(2 \pi)^{2 n}} \int \sigma(x, \xi)\left(\begin{array}{c}
\hat{w}(\xi) \\
\hat{v}(\xi)
\end{array}\right) e^{\jmath \sum_{i} x^{i} \xi_{i}} d \xi
$$

where $\hat{w}(\xi), \hat{v}(\xi)$ denote the Fourier transforms of $w_{\theta(x)}$ $v_{\theta(x)}$, respectively, in the local coordinate patch, and $\sigma$ is the formal symbol,

$$
\begin{aligned}
& \sigma(x, \xi)= \\
& \left(\begin{array}{cc}
A_{\theta(x)}-\jmath \sum_{i} f^{i}(\theta(x)) \xi_{i} & -B_{\theta(x)} B_{\theta(x)}^{T} \\
-G_{\theta(x)} & -A_{\theta(x)}^{T}-\jmath \sum_{i} f^{i}(\theta(x)) \xi_{i}
\end{array}\right)
\end{aligned}
$$

where $\xi_{i}$ stands, formally, for $-\jmath \frac{\partial}{\partial x^{i}}$. Globally, $P_{\sigma}$ is defined as

$$
P_{\sigma}: \Gamma\left(\Theta, T \Theta \oplus T^{*} \Theta\right) \rightarrow \Gamma\left(\Theta, T \Theta \oplus T^{*} \Theta\right)
$$

where $\Gamma$ denotes smooth sections. The symbol is defined, intrinsically, as

$$
\sigma: T^{*} \Theta \rightarrow \operatorname{hom}\left(T \Theta \oplus T^{*} \Theta, T \Theta \oplus T^{*} \Theta\right)
$$

Observe that $P_{\sigma}$ is certainly not elliptic. However, what makes its Fredholmness analysis easy is the following:

Theorem 9 If $A_{\theta}$ does not have eigenvalues on the imaginary axis, the symbol $\sigma$ is invertible.

Recall that $P_{\sigma}: \Gamma \rightarrow \Gamma$ is Fredholm iff both ker $P_{\sigma}$ and coker $\left(P_{\sigma}\right)$ are finite dimensional. The formal adjoint of the operator $P_{\sigma}, P_{\sigma}^{*}$, is defined as the operator such that $\left(a, P_{\sigma} b\right)=\left(P_{\sigma}^{*} a, b\right)$ where

$$
(a, b)=\int_{\Theta}\langle a(\theta), b(\theta)\rangle \mu(d \theta)
$$

for some measure $\mu$ with smooth density [12, page 26]. With these concepts, the Fredholm index is defined as

$\operatorname{dim} \operatorname{ker} P_{\sigma}-\operatorname{dim}$ coker $P_{\sigma}=\operatorname{dim} \operatorname{ker} P_{\sigma}-\operatorname{dim} \operatorname{ker} P_{\sigma}^{*}$

Using the composition of symbols, it is easily seen that $P_{\sigma} P_{\sigma^{-1}}-I$ and $P_{\sigma^{-1}} P_{\sigma}-I$ are smoothing operators, that is, integral operators with smooth kernels, which are known to be compact [12], [6]. Therefore, by Atkinson's theorem, $P_{\sigma}$ is Fredholm. The same applies to $P_{\sigma}-\lambda I$, provided $A_{\theta}-\lambda I$ does not have eigenvalues on the imaginary axis.

We now look at the index of the operator and make a merger with ergodic theory.

Lemma 10 If the partial differential operator $\sum_{i} f^{i} \frac{\partial}{\partial x^{i}}$ is formally skew self-adjoint, viz.,

$$
\sum_{i} f^{i}(\theta(x)) \frac{\partial}{\partial x^{i}}+\left(\sum_{i} f^{i}(\theta(x)) \frac{\partial}{\partial x^{i}}\right)^{*}=0
$$

then the operator $P_{\sigma}$ is Hamiltonian, viz., $J P_{\sigma} J=P_{\sigma}^{*}$ where $J=\left(\begin{array}{cc}0 & I \\ -I & 0\end{array}\right)$ 
Theorem 11 If either of the following conditions holds

1. $\Theta$ is a compact subset of $\mathbb{R}^{n}$ and $f \mid \partial \Theta=0$,

2. or $\Theta$ is a compact, smooth, orientable ${ }^{3}$ manifold, viz., $\partial \Theta=\emptyset$,

and if the flow $f$ has an invariant measure $\mu(d \theta)$ absolutely continuous relative to the volume form $d x^{1} \cdots d x^{n}$ and with a smooth Radon-Nikodym derivative, viz, $\operatorname{div}(\nu f)=\sum_{i} \frac{\partial \nu f^{i}}{\partial x^{i}}=0$, where $\nu(\theta)$ is the Radon-Nikodym derivative [7, Chapter 1, Section 3], $\nu(\theta)=\frac{\mu(d \theta)}{d x^{1} \cdots d x^{n}}$, then the operator $\sum_{i} f^{i} \frac{\partial}{\partial x^{i}}$ is skew self-adjoint.

Proof: The proof basically relies on proving (7) by an integration of the type (6), converted to an integration of an exterior differential form over the subset $\Theta$ of $\mathbb{R}^{n}$ or over all coordinate patches across the whole manifold $\Theta$, and proving, using Stokes' theorem, that it vanishes. $\square$

Theorem 12 If the operator $P_{\sigma}$ is Fredholm and Hamiltonian, then its analytical index vanishes, viz., $\operatorname{dim}$ ker $P_{\sigma}-\operatorname{dim}$ coker $P_{\sigma}=0 . \square$

We now turn our attention to the operator $P_{\sigma-\lambda I}$. The operator $P_{\sigma-\lambda I}, \lambda \neq 0$, is not Hamiltonian anymore because indeed, $J P_{\sigma-\lambda I} J \neq\left(P_{\sigma-\lambda I}\right)^{*}$. Therefore, depending on $\lambda$, the operator $P_{\sigma-\lambda I}$ could have nonvanishing index and hence have a kernel - even when $\operatorname{det}(\sigma-\lambda I) \neq 0$.

Theorem 13 There exists an homomorphism

$$
\begin{array}{rll}
h & : \quad\left[\Theta \times \mathbb{R}^{2 n}: \mathbb{C} \backslash\{0+j 0\}\right] \rightarrow \mathbb{Z} \\
\operatorname{deg}(\sigma-\lambda I) & \mapsto \quad \operatorname{index}_{a}\left(P_{\sigma-\lambda I}\right)
\end{array}
$$

between the set of homotopy classes of maps $\sigma(\theta, \xi)-$ $\lambda_{\theta} I: \Theta \times \mathbb{R}^{2 n} \rightarrow \mathbb{C} \backslash\{0+j 0\}$ and the connected components of the set $\mathcal{F}(\Gamma, \Gamma)$ of Fredholm operators, each connected component being uniquely determined by its Fredholm index.

Clearly, since $\mathbb{R}^{2 n}$ is contractible, the degree of the map $\Theta \times \mathbb{R}^{2 n} \rightarrow \mathbb{C} \backslash\{0+\not 0\}$ can be reduced to the degree of $\sigma(\cdot, \xi)-\lambda I: \Theta \rightarrow \mathbb{C} \backslash\{0+\jmath 0\}$ at constant $\xi$. Since $\mathbb{C} \backslash\{0+j 0\}$ is contractible to a circle, the set of allowable homotopy classes are those of the induced map $\Theta \rightarrow S^{1}$, isomorphic to cohomology group $H^{1}(\Theta, \mathbb{Z})$ by the Hopf theorem.

\footnotetext{
${ }^{3}$ Orientability is required for the volume form $d x^{1} \cdots d x^{n}$ to be expressible as an exterior differential form $d x^{1} \wedge \cdots \wedge d x^{n}$ of constant sign across the manifold.
}

Theorem 14 There exists a homomorphism

$$
\begin{array}{rll}
h & : & H^{1}(\Theta) \rightarrow \mathbb{Z} \\
\operatorname{deg}(\sigma-\lambda I) & \mapsto & \text { index }_{a}\left(P_{\sigma-\lambda I}\right.
\end{array}
$$

\section{References}

[1] R. L. Bishop and S. I. Goldberg. Tensor Analysis on Manifolds. Dover, 1980.

[2] S. Bohacek and E. A. Jonckheere. Linear dynamically varying $H_{\infty}$ control of chaos. In $\mathrm{H}$. Nijmeijer and A. J. van der Schaft, editors, NOLCOS98, Nonlinear Control Systems Design Symposium, pages 745-750, University of Twente, Enschede, The Netherlands, July 1-3 1998. IFAC, Elsevier.

[3] A. Hammad, E. Jonckheere, C.-Y. Cheng, S. Bhajekar, and C.-C. Chien. Stabilization of chaotic dynamics: a modern control approach. International Journal of Control, 64(4):663-677, 1996.

[4] E. Jonckheere, J.C. Juang, and L.M. Silverman. Spectral theory of the linear quadratic and H-infinity problems. Linear Algebra and its Applications, 1989.

[5] E. A. Jonckheere and L. M. Silverman. The linear-quadratic optimal control problem-the operator theoretic viewpoint. Operator Theory, Advances and Applications, 12:277-302, 1984. Birkhäuser.

[6] R. S. Kulkarni. Index theorems of AtiyahBott-Patodi and curvature invariants. Séminaire de Mathématiques Supérieures, Département de Mathématiques-Université de Montréal, C. P. 6128, Montréal, Canada, 1975.

[7] R. Ma né. Ergodic Theory and Differential Dynamics. Springer-Verlag, 1987.

[8] B. O'Neill. Elementary Differential Geometry. Academic Press, 1997.

[9] L. S. Pontryagin. Topological Groups (Second Edition). Gordon and Breach, 1966.

[10] M. Rathinam and R. M. Murray. Configuration flatness of Lagrangian systems underactuated by one control. In Proceedings of the 35th Conference on Decision and Control, pages 1688-1693, Kobe, Japan, December 11-13 1996. IEEE.

[11] R. W. Sharpe. Differential Geometry - Cartan's Generalization of Klein's Erlangen Program. SpringerVerlag, 1997.

[12] M. A. Shubin. Pseudodifferential Operators and Spectral Theory. Springer-Verlag, Berlin, Heidelberg, New York, 1987.

[13] J. J. Stocker. Differential Geometry. Wiley Classics Library, 1989. 